For review for publication in IEEE Transactions on Aerospace and Electronic Systems

\title{
ROBUST DAMAGE-MITIGATING CONTROL OF AIRCRAFT FOR HIGH PERFORMANCE AND STRUCTURAL DURABILITY ${ }^{\ddagger}$
}

\author{
Jeffrey Caplin \\ Asok Ray ${ }^{\dagger}$, Senior Member, IEEE \\ Mechanical Engineering Department \\ The Pennsylvania State University \\ University Park, PA 16802 \\ Suresh M. Joshi, Fellow, IEEE \\ NASA Langley Research Center \\ Hampton, VA 23681-0001 \\ † Corresponding Author: Tel: (814) 865-6377; Email: axr2(i)psu.edu \\ Keywords: Damage-Tolerant Systems, Robust Control, Structural Durability
}

\begin{abstract}
This paper presents the concept and a design methodology for robust damage-mitigating control (DMC) of aircraft. The goal of DMC is to simultaneously achieve high performance and structural durability. The controller design procedure involves consideration of damage at critical points of the structure, as well as the performance requirements of the aircraft. An aeroelastic model of the wings has been formulated and is incorporated into a nonlinear rigid-body model of aircraft flight-dynamics. Robust damage-mitigating controllers are then designed using the $\mathrm{H}_{\infty}$-based structured singular value $(\mu)$ synthesis method based on a linearized model of the aircraft. In addition to penalizing the error between the ideal performance and the actual performance of the aircraft, frequencydependent weights are placed on the strain amplitude at the root of each wing. Using each controller in turn, the control system is put through an identical sequence of maneuvers, and the resulting (varying amplitude cyclic) stress profiles are analyzed using a fatigue crack growth model that incorporates the effects of stress overload. Comparisons are made to determine the impact of different weights on the resulting fatigue crack damage in the wings. The results of simulation experiments show significant savings in fatigue life of the wings while retaining the dynamic performance of the aircraft.
\end{abstract}

¥The research work reported in this paper has been supported in part by:

NASA Langley Research Center under Grant No. NCC-1-249;

National Science Foundation under Grant Nos. DMI-9424587 and CMS-9531835;

National Academy of Sciences under a Research Fellowship award to the second author. 


\section{INTRODUCTION}

In the post-Cold War era, there is an increasing concern about high acquisition and maintenance cost associated with complex weapons systems. Fighter aircraft is a good example of such systems because the airframe undergoes significant cyclic stresses resulting in the need for frequent inspection and replacement of critical components. While airframe manufacturers are constantly updating the technology base to handle higher stress levels, these improvements often do not fully translate into an equivalent increase in component life due to the ever-increasing performance requirements of fighter aircraft. This is particularly true of the current generation of fighter aircraft, as the high thrust-to-weight ratio allows extreme flight maneuvers that were not possible earlier and thereby the airframe is often subjected to very high instantaneous and sustained stress levels.

From an economic standpoint it is desirable to obtain the maximum amount of useful life from the most expensive (and hard to replace) components of the aircraft, as well as to reduce the number of maintenance inspections required to ensure structural integrity of critical components. This practice is also desirable from an operational viewpoint, since reductions in downtime for inspection and repair result in increased availability. However, since failure of certain components may result in loss of the aircraft, and more importantly, loss of human life, safety considerations mandate replacement of all critical components before a failure is likely to occur. This requirement is realized in the following way. A fighter aircraft that exceeds its design load factor during a flight is temporarily removed from service, and it must undergo a rigorous inspection to determine if any special maintenance is required prior to its return to flying status. (Note: Load factor is defined as the total lift force acting on the aircraft divided by the aircraft weight).

Designers of flight control systems have recognized the possibility of actively reducing damage in certain aircraft structures, particularly the wing. The simplest concept that is currently employed on both F-16 and F/A-18 aircraft is the so-called "g-limiter". It serves to limit the aircraft's maximum load factor to a predefined value. Transport aircraft have used the Gust Load Alleviation (GLA) system [Matsuzaki et al., 1989; Baldelli et al, 1993] that uses feedback from accelerometers on the wing to drive special control surfaces in order to reduce the additional loads imposed by atmospheric disturbances. A similar concept, known as Maneuver Load Alleviation (MLA) [McLean, 1990] or Maneuver Load Control (MLC) [Thornton, 1993], has been proposed for high-performance aircraft. The aim of these systems is to shift the lift distribution inboard during high loading conditions to limit the bending moment at the wing root. Dynamic stresses have been considered in the so-called Fatigue Reduction (FR) system [McLean, 1990] that seeks to minimize the amplitude and/or number of stress cycles experienced at the critical point(s). While these systems have shown tangible benefits, there is apparently a common weakness that may well prevent them from achieving their maximum potential. In all cases, the actual dynamics of the fatigue crack damage phenomenon in the structural material are not included in the analysis. It is simply assumed that, by limiting the peak stress at the critical points of the structure, life-savings could be maximized. Since transient stress overloads could result in a temporary retardation of crack growth [Anderson, 1995; Patankar et al., 1999], the frequency content of the applied stresses could be shaped by control actions to achieve larger fatigue life than the traditional approach of simply limiting the peak stress. 
This paper addresses the above issue focusing on fatigue damage mitigation in the wings of high performance aircraft that are usually instrumented for health monitoring and control. The thrust of the paper is on robust damagemitigating control (DMC) where the goal is to achieve large gains in structural durability by manipulation of stress profiles with no significant loss of performance [Ray et al., 1994]. This concept of DMC has been investigated for reusable rocket engines [Dai and Ray, 1996; Holmes and Ray, 1998], rotorcraft [Rozak and Ray, 1997, 1998], and fossil fuel power plants [Kallappa et al., 1997]. In all cases, simulation results show a substantial increase in component life with no significant loss in system performance. Efficacy of the DMC concept has also been demonstrated by laboratory experimentation on test apparatuses [Tangirala et al., 1998; Zhang and Ray, 1998].

The paper is organized in five sections including the introduction. Section 2 describes model formulation for damage mitigating controller design. Section 3 presents a procedure for synthesis of the robust damage-mitigating control law. Section 4 presents the overall simulation structure and the results of the aircraft performance and crackgrowth damage for a family of robust controllers. Section 5 summarizes and concludes the paper with recommendations for future work.

\section{Model formulation for Damage-mitigatng CONTROller Design}

Although it has long been recognized that controller design for highly flexible aircraft, such as transports, requires dynamic models that explicitly include structural flexibility [McLean, 1990], these effects are often ignored on aircraft that experience relatively small elastic deformation. This is particularly true when modern robust control techniques, such as $\mathrm{H}_{\infty}$-synthesis, are employed, since the effects of unmodeled dynamics due to flexibility can be included within the (unstructured) uncertainty model. However, explicit modeling of structural flexibility may provide a solution to another problem faced by flight control systems designers, namely that of control surface redundancy. High-performance aircraft require two (or more) different sets of control surfaces for roll control and, in some cases, have multiple controls for pitch and yaw as well. As these controls have different levels of effectiveness at different flight conditions, they appear to be redundant actuators in the controller synthesis process at a given operating condition. Therefore, special measures are required to allocate commands between the various control surfaces. Methods that have been developed to date include the use of a nonlinear control selector [Buffington et al., 1994], or the use of off-line constrained optimization procedures [Durham, 1992]. In both cases, the methods only examine the effects of the controls on the rigid-body motion of the aircraft. The damage mitigating control (DMC) takes advantage of the control surface redundancy by utilizing the elastic behavior of the aircraft structure as well as the rigid-body motion. The theme of DMC design is that different locations of control surfaces on the airframe may result in different effects on the elastic modes of the aircraft structure. Thus, the control systems designer can make use of all available control surfaces to simultaneously achieve the desired level of performance while mitigating the structural damage by reshaping the stress profile.

So far the only application of DMC to aviation systems has been the work of Rozak and Ray $(1997,1998)$ who developed a robust controller for rotorcraft with the objective of reducing damage to the control horn of the main rotor. Since the control horn does not directly experience any significant aerodynamic forces, the loading is of a 
purely mechanical nature. In contrast, the loads (and hence stresses) acting on an aircraft wing occur due to aeroelasticity, which deals with interactions of aerodynamic forces with flexible structures. The aerodynamic forces play a dominant role in determining the dynamics of the aircraft that, in turn, lead to deformations and stresses in the critical structures. Therefore, changes in aerodynamic forces due to structural deformation have been included in the plant dynamic model in combination with the rigid body model.

\subsection{The Rigid Body Model}

The model describes the motion of a rigid-aircraft of fixed mass flying through a stationary atmosphere over a flat, non-rotating earth. The aircraft is a generic, twin engine, high-performance fighter similar to the F-15. Three coordinate systems that are used together to define the position and orientation of the aircraft are the inertial axes, the vehicle-carried vertical axes, and the body-fixed axes. As the name implies, the inertial axes are considered fixed in space, with the $\mathrm{X}$-axis directed north, the $\mathrm{Y}$-axis directed east, and the $\mathrm{Z}$-axis directed down. The vehicle carried vertical axes (denoted by the subscript ' $v$ ') have the same orientation as the inertial axes, but their origin lies at the aircraft center of gravity, and translates with the aircraft. Three state variables are needed to define the position of the vehicle-carried vertical axes with respect to the inertial axes. These are the displacement north, displacement east, and the altitude, denoted as $x, y$, and $h$, respectively.

The body-fixed axes (denoted by the subscript ' $b$ ') also have their origin at the aircraft center of gravity; however, they rotate as well as translate with the body. They are oriented such that the $\mathrm{x}_{\mathrm{b}}$-axis is positive towards the nose of the aircraft, the $y_{b}$-axis is positive towards the right wing tip, and the $z_{b}$-axis is positive towards the bottom of the aircraft. The orientation of the body-fixed axes (3-2-1 rotation) with respect to the vehicle-carried vertical axes is defined by the state variables $\Psi, \Theta$, and $\Phi$, known respectively as the yaw angle, pitch angle, and roll (or bank) angle. The order in which these rotations are carried out is demonstrated in Figure 1. First, the vehiclecarried vertical axes are rotated about the $z_{\mathrm{v}}$-axis through the angle $\Psi$ to obtain an intermediate set of axes designated $\left(x_{1}, y_{1}, z_{1}\right)$. This axis system is then rotated about the $y_{1}$-axis through the angle $\Theta$ to obtain the $\left(x_{2}, y_{2}, z_{2}\right)$ axes. Finally, this system is rotated about the $x_{2}$-axis through the angle $\Phi$ to obtain the body-fixed axes.

Two coordinate systems are used to define the relative motion between the aircraft and the atmosphere. The stability axes (denoted by the subscript ' $s$ ') are obtained by rotating the body-fixed axes through the angle of attack $\alpha$, which is the angle between the $x_{b}$-axis and the projection of the velocity vector onto the $x_{b}-z_{b}$ plane. The pitching behavior of the body-fixed axes with respect to the stability axes is used to characterize the longitudinal stability of the aircraft, hence the name "stability axes."

The wind axes (denoted by the subscript ' $w$ ') are obtained by rotating the $z_{s}$-axis through the sideslip angle $\beta$, which brings the $x_{w}$-axis in line with the velocity vector. The primary advantage of using the wind axes, and choosing the magnitude of the total velocity vector as one of the state variables, is that this approach permits the explicit computation of derivatives of $\alpha$ and $\beta$ in terms of other state variables. An alternate formulation, which used the velocity components along the body-fixed axes as states, would result in equations for the derivatives of $\alpha$ 
and $\beta$ in terms of both the remaining states and their derivatives [Blakelock, 1991]. Figure 2 shows the relationship between the body-fixed, stability, and wind axes. The last three state variables define the rotational motion of the aircraft. The roll, pitch, and yaw rates, denoted as p, $\mathrm{q}$, and $\mathrm{r}$, respectively, are the rotational rates about the $\mathrm{x}_{\mathrm{b}}$-axis, $y_{b}$-axis, and $z_{b}$-axis, respectively. It should be noted that while the directions of these rotational rates are defined by the orientation of the body-fixed axes, their magnitudes are measured in the inertial reference frame.

The rigid-body flight dynamic model in this paper are similar to the corresponding model developed for the AIAA Controls Design Challenge [Brumbaugh, 1991] and therefore these model equations not repeated here. The control surfaces include left and right ailerons, left and right stabilators, and a single rudder. Although the aircraft has five control surfaces, only four variables are required to specify their positions: the aileron deflection $\delta_{A}$; the rudder deflection $\delta_{R}$, the symmetric stabilator deflection $\delta_{H}$; and the differential stabilator deflection $\delta_{D}$. The positions of the individual control surfaces are determined as follows:

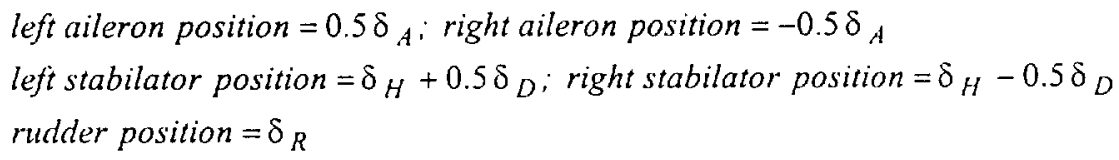

In the original model of Brumbaugh (1991), all actuator dynamics are represented as first order lags with a time constant of $50 \mathrm{~ms}$ that has been replaced with more detailed dynamics [Adams et al., 1994]. The transfer functions and rate limits of the actuators are given in Table I.

Linearization of the equations of motion for a rigid, fixed-wing aircraft yields two uncoupled sets of equations. One set governs the longitudinal dynamics of the aircraft while the other governs the lateral dynamics. We have followed the standard practice to design separate controllers for lateral and longitudinal dynamics based on the uncoupled linearized models and then evaluate the control system based on the simulation of the nonlinear model.

The stick-fixed longitudinal motion of a rigid aircraft disturbed from equilibrium flight is described by two oscillatory modes of motion: the short period mode and the long period (or phugoid) mode. The short period mode typically has a period on the order of a few seconds, with motion characterized by changes in angle of attack, pitch angle, and altitude, while the flight velocity remains practically constant. The phugoid mode has a much longer period, on the order of tens or hundreds of seconds, with motion characterized by changes in velocity, pitch angle, and altitude, with angle of attack remaining approximately constant. Because of the slow dynamics associated with the phugoid mode, this mode has been ignored during the synthesis of the damage-mitigating control laws for manual flight. The pitch rate $q$, and angle of attack $\alpha$ are the state variables of interest for longitudinal rigid body motion. However, for the design of an autopilot, the phugoid motion becomes the primary mode of interest.

The desired short period response of the aircraft is that of a second order system. The natural frequency is a function of the acceleration sensitivity of the aircraft, which is the change in load factor per unit change in angle of attack. The acceleration sensitivity is determined by the aerodynamics of the aircraft and the particular flight condition under consideration. Thus, the desired natural frequency also varies with different flight conditions. The 
damping ratio of the short period mode is required to be between 0.35 and 1.3 for all flight conditions based on MIL-F-8785C specifications [DoD, 1980].

The roll rate $p$, yaw rate $r$, and sideslip angle $\beta$ are the state variables of interest for lateral rigid body motion. The stick-fixed lateral motion of a rigid aircraft is described by three natural modes: (i) the Dutch roll mode consisting of lightly damped, oscillatory, out-of-phase roll, yaw, and sideslip motions; (ii) the roll mode consisting of a non-oscillatory, highly convergent mode describing the rolling characteristics of the aircraft; and (iii) the spiral mode consisting of non-oscillatory, convergent or divergent motion following a sideslip disturbance. Note that an unstable spiral mode may cause the aircraft to go into a turn that becomes increasingly tighter with time. The handling qualities requirements specify that the Dutch roll mode should have a frequency of at least 1 radian per second. The damping ratio must be greater than or equal to 0.4 , or the product of the frequency and damping ratio should be greater than or equal to 0.4 radians per second, whichever results in the larger value for the required damping. The roll mode requirement states that the roll time constant must be less than or equal to 1.0 second. This requirement is actually conservative with regards to modern fighters, which typically have roll time constants in the range of 0.33 to 0.5 seconds [Adams et al., 1994]. The spiral mode requirement specifies that the minimum time to reach a $40^{\circ}$ bank angle following a $20^{\circ}$ bank angle disturbance must be greater than or equal to 12 seconds. Because of the slower dynamics of the spiral mode, it is typically ignored during controller synthesis.

\subsection{Atmospheric Model}

Atmospheric properties are based on the US Standard Atmosphere (1962). The model outputs values for temperature, static pressure, density, and speed of sound as functions of altitude. While not strictly an atmospheric property, the model also includes tabulated values for the acceleration due to gravity, also as a function of altitude. The atmospheric model in this paper is similar to the corresponding model developed for the AIAA Controls Design Challenge [Brumbaugh, 1991] and is not repeated here.

\subsection{Aeroelastic Model}

Aerodynamic forces acting on a body depend on the time history of the body's motion [Etkin, 1972]. When the dynamics of a rigid aircraft are the subject of interest, particularly if the aircraft does not perform any severe maneuvers, the much faster dynamics of the flowfield are ignored based on the principle of singular perturbation. For aircraft that are required to perform extreme maneuvers, it is often sufficient to introduce approximate correction factors into the equations of motion to account for any unsteady aerodynamic forces. These correction factors generally depend on the time derivatives of angle of attack and sideslip angle. However, when the flexible structures of aircraft (that have a faster time scale than the rigid-body dynamics) are of interest, it becomes necessary to explicitly model the dynamics of the flow-field to identify any potential instability due to fluid-structure interactions, known as flutter. Since wing flutter results in catastrophic failure of the aircraft, it is prevented either through the design of the wing, or through the use of Active Flutter Suppression (AFS). Therefore, an aeroelastic model of the critical structure (i.e., the wings) is required for the DMC design for the following reasons: 
- To shape the profiles of transient stresses for fatigue damage reduction; and

- To ensure that the control system does not adversely affect the flutter characteristics.

\subsubsection{Structural Model}

Although composite wing structures have been used in recent aircraft, most fighter aircraft have wings that are at least partially, and in most cases solely, built from ductile alloys. A typical wing structure contains at least two spars that run the length of the wing semi-span to bear the majority of the bending loads. The spars and the skin together form several torsion boxes to resist twisting deformation of the wings. The wings also contain many lesser structural members whose primary function is to maintain the shape of the skin. In this paper, the structural model is formulated as a pair of Euler beams to represent the important structural behavior of the wings. Each beam model is aligned with the elastic axis (i.e. the line through which loads applied normal to the plane of the wing result in pure bending). The center portion of the model, where the beams meet, is assigned proportionately higher values of bending and torsional stiffness in order to represent the fuselage. The model is spatially discretized and cast in the finite element setting. While the details of the finite-element model, including the element type and shape functions, are reported by Caplin (1998), its basic features and dominant mode shapes are presented below:

The generalized displacement vector $\xi(t)$ is obtained by orthogonal transformation of the physical displacement vector $\theta(t)$, i.e., $\xi(t)=\Phi^{T} \theta(t)$ where $\Phi$ is the orthogonal matrix whose columns are the individual mode shapes. The governing equation for $\xi(t)$ is obtained in the transformed coordinates as:

$$
M \ddot{\xi}(t)+C \dot{\xi}(t)+K \xi(t)=f(t)
$$

where the transformed "modal mass" and "modal stiffness" matrices, $M$ and $K$, are diagonal; $C$ is the "modal damping" matrix representing energy dissipation; and $f(t)$ the total generalized force vector which is obtained by orthogonal transformation of the applied nodal force vector that is a linear combination of aerodynamic force due to both vibratory motion of flexible modes and rigid-body motion. Accordingly, the total generalized force vector is expressed as:

$$
f(t)=f_{\text {flex }}(t)+f_{\text {rigid }}(t)
$$

where $f_{f l e x}$ is the generalized aerodynamic force vector acting on the flexible modes and $f_{\text {rigid }}$ is the generalized force vector due to rigid-body motion.

For the specific wing structure considered in this paper, we have used the six lowest modes, of which three are symmetric and three are antisymmetric with respect to the aircraft body-fixed x-axis. Figures 3 and 4 show the symmetric mode shapes and the antisymmetric mode shapes for both linear displacement and angular twist. It is necessary to make the distinction between symmetric and antisymmetric modes because the dynamics of the two sets of modes are uncoupled from each other. Within each set, however, the dynamics are coupled due to the aerodynamic forces generated by the deformation of the wing. 


\subsubsection{Integrated Model of Unsteady Aerodynamics and Structural Dynamics}

Although the current state-of-the-art in Computational Fluid Dynamics (CFD) allows time-domain solutions to the Euler equations (for inviscid compressible flow) or the Navier-Stokes equations (for viscous compressible flow) for many flows of practical interest, the use of these techniques is still rather limited due to high computational cost. Within the aerospace industry, a vast majority of unsteady flow applications, such as flutter analysis, rely on computational techniques that have been developed for the restricted case of thin wings undergoing simple harmonic motion for unsteady subsonic potential flow [Dowel et al., 1995]. The Doublet-Lattice Method [Albano and Rodden, 1969] has been adopted in this paper for aerodynamic analysis. In this method, the wing is divided into a finite number of trapezoidal panels. The lifting force acting on each panel is assumed to be concentrated along the one-quarter chord line of the panel, where a line of acceleration potential doublets is placed. The strength of the doublets is assumed to be uniform within each panel and is allowed to vary from one panel to the next. A control point is placed at the three-quarter-chord point of the mid-span of each panel. Details are reported by Caplin (1998).

The transfer matrix $Q$ from the generalized displacement vector $\xi$ to the flexible part $f_{f l e x}$ of the total generalized force vector $f$ is approximated as a function of the dimensionless Laplace transform variable $\bar{s}$ and dynamic pressure $\bar{q}$ \{Karpel, 1990]:

$$
\mathrm{Q}(s) \approx\left(A_{0}+\bar{s} A_{1}+\bar{s}^{2} A_{2}+\bar{s} D(\bar{s} \mathrm{I}-\mathrm{R})^{-1} E\right) \bar{q} ; \quad \bar{s} \equiv \frac{s b}{U_{\infty}} ; \quad \vec{q} \equiv \frac{1}{2} \rho_{\infty}\left(U_{\infty}\right)^{2}
$$

where $U_{\infty}$ and $\rho_{\infty}$ are the undisturbed free-stream air flow speed and density, respectively; $b$ is the wing semispan; the matrix $A_{0}$ is obtained from the steady-sate response of experimental or CFD simulation data; other matrices, $A_{1}, A_{2}, D$, and $E$, are obtained by frequency-domain system identification based on experimental or CFD simulation data; and $R$ is a diagonal matrix whose elements ate chosen to be the poles of additional aerodynamic states within the frequency range of interest.

Remark 2.1: The terms involving the matrices $A_{0}, A_{1}$, and $A_{2}$ in Eq. (3) capture the dependence of the aerodynamic forces on the displacement, velocity, and acceleration, respectively, of the wing mode shapes. The remaining term, involving the matrices $D, R$, and $E$ on the right hand side of Eq. (3), account for the lag in aerodynamic forces.

Making the transformation of Eq. (3) into the time domain and substituting the resulting expression into Eqs. (1) and (2) yield the following set of ordinary differential equations:

$$
\begin{aligned}
& M \ddot{\xi}(t)+C \dot{\xi}(t)+K \xi(t)=\left(\mathrm{A}_{0} \xi(t)+\frac{b}{U_{\infty}} \mathrm{A}_{1} \dot{\xi}(t)+\left(\frac{b}{U_{\infty}}\right)^{2} \mathrm{~A}_{2} \ddot{\xi}(t)+D \mathrm{x}_{a}(t)\right) \bar{q}+f_{\text {rigid }}(t) \\
& \dot{x}_{a}(t)=E \dot{\xi}(t)+\frac{U_{\infty}}{b} R x_{a}(t)
\end{aligned}
$$


where $x_{a}$ is the vector of selected states to represent the aerodynamic lag; and $f_{\text {rigid }}$ is the part of the total generalized force vector contributed by the rigid-body motion as defined in Eq. (2).. The aeroelastic model in Eq. (4) is rewritten in the state space setting for synthesis of damage-mitigating controllers as:

$$
\begin{aligned}
& \frac{d}{d t}(\xi(t))=\dot{\xi}(t) \\
& \frac{d}{d t}(\dot{\xi}(t))=\bar{M}^{-1}\left(-\overline{K \xi}(t)-\bar{C} \dot{\xi}(t)+\bar{q} D x_{a}(t)+f_{\text {rigid }}(t)\right) \\
& \frac{d}{d t}\left(x_{a}(t)\right)=\mathrm{E} \dot{\xi}(t)+\frac{U_{\infty}}{b} R x_{a}(t)
\end{aligned}
$$

by introducing the following definitions:

$$
\bar{M} \equiv M-\left(\frac{b}{U_{\infty}}\right)^{2} \bar{q} A_{2} ; \quad \overline{\mathrm{C}} \equiv \mathrm{C}-\frac{b}{U_{\infty}} \bar{q} A_{1} ; \quad \bar{K} \equiv K-\bar{q} A_{0}
$$

The aeroelastic model in Eq. (5) forms two uncoupled sets of equations, one for the symmetric modes and the other for the antisymmetric modes. Additional aerodynamic terms representing $f_{\text {rigid }}$ in Eq. (5) must be added to each model to account for the relevant rigid-body motions. Furthermore, the perturbations in the rigid-body aerodynamic coefficients are different for each model. These considerations are addressed in the next two sections.

\subsubsection{Symmetric Aeroelastic Model}

In general, the largest contribution to the aerodynamic loads acting on the wing is due to the rigid-body angle of attack. Strictly speaking, this requires the addition of three terms to Eq. (5); one for the angle of attack, and one each for its first two derivatives. However, it is generally recognized that terms involving the second derivative of the rigid body motion may be neglected for characteristic frequencies below $2 \mathrm{~Hz}$ [Pierce (1988)]. Since angle of attack $\alpha$ does not have any significant frequency content above $2 \mathrm{~Hz}$, the term involving the acceleration $\ddot{\alpha}$ has not been included in the complete aeroelastic model.

Two inertial terms, $Q_{a z} a_{z}(t)$ and $Q_{\dot{q}} \dot{q}(t)$, which are proportional to normal acceleration and pitch acceleration of the aircraft, respectively, are added to the model when longitudinal rigid-body motion is considered. Substituting these inertial terms along with additional aerodynamic terms into Eq. (5) yields the following set of equations for the symmetric aeroelastic model:

$$
\begin{aligned}
& \frac{d}{d t}(\xi(t))=\dot{\xi}(t) \\
& \frac{d}{d t}(\dot{\xi}(t))=\overline{\mathrm{M}}^{-1}\left(-\overline{\mathrm{K}} \xi(t)-\overline{\mathrm{C}} \dot{\xi}(t)+\left(A_{\alpha} \alpha(t)+\frac{b}{U_{\infty}} A_{\dot{\alpha}} \dot{\alpha}(t)+\mathrm{D} x_{a}(t)\right) \bar{q}+Q_{a z} a_{z}(t)+Q_{\dot{q}} \dot{q}(t)\right)(7) \\
& \frac{d}{d t}\left(x_{a}\right)=E \dot{\xi}(t)+\frac{U_{\infty}}{b} R x_{a}(t)+E_{\dot{\alpha}} \dot{\alpha}(t)
\end{aligned}
$$

The main effect of symmetric deformation of the wing on the overall dynamics of the aircraft is assumed to be due to the change in lift coefficient. The transfer function from generalized displacement to change in lift 
coefficient is obtained in the same manner as the transfer function from generalized displacement to generalized force. Thus the unsteady perturbation in lift coefficient is obtained in the time-domain as:

$$
\Delta C_{L}(t)=\mathrm{A}_{0_{C_{L}}} \xi(t)+\frac{b}{U_{\infty}} \mathrm{A}_{1_{C_{L}}} \dot{\xi}(t)+\left(\frac{b}{U_{\infty}}\right)^{2} \mathrm{~A}_{2_{C_{L}}} \ddot{\xi}(t)+D_{C_{L}} x_{a}(t)
$$

\subsubsection{Antisymmetric Aeroelastic Model}

Two additional sets of aerodynamic terms are added to the antisymmetric model. First, deflection $\delta_{A}(t)$ of the ailerons is viewed as an additional deformation mode of the wing, and thus additional aerodynamic terms involving aileron position, rate, and acceleration must be included. Second, terms involving the rigid-body roll rate and roll acceleration are required although roll angle has no effects. Only one inertial term, $Q_{\dot{p}} \dot{p}(t)$, which is proportional to roll acceleration of the aircraft, is added to the model when lateral rigid-body motion is considered. The antisymmetric aeroelastic model thus takes the following form:

$$
\begin{aligned}
& \frac{d}{d t}(\eta(t))=\dot{\eta}(t) \\
& \frac{d}{d t}(\eta(t))=\bar{M}^{-1}\left(-\bar{K} \eta(t)-\bar{C} \eta(t)+\bar{q} D y_{a}(t)+Q_{\dot{p}} \dot{p}(t)\right)
\end{aligned}
$$

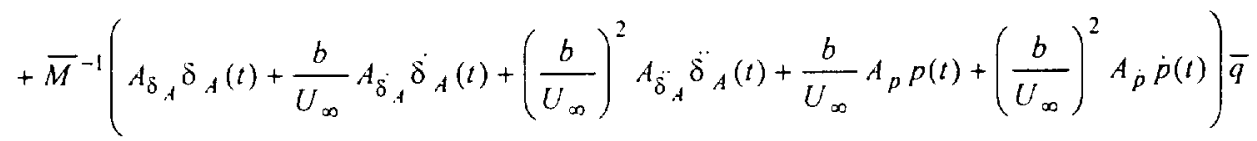

$$
\begin{aligned}
& \frac{d}{d t}\left(y_{a}\right)=E \dot{\eta}(t)+\frac{U_{\infty}}{b} R y_{a}(t)+E_{\dot{\delta}_{A}} \dot{\delta}_{A}(t)+E_{p} p(t)
\end{aligned}
$$

where the symbols $\eta$ and $y_{a}$ have been used for the generalized displacement vector and the aerodynamic state, respectively, in order to distinguish the antisymmetric states from the symmetric states $\xi$ and $x_{a}$. The main effect of antisymmetric deformation of the wing on the overall dynamics of the aircraft is due to the change in roll moment coefficient. Its transfer function with respect to generalized displacement is obtained similar to Eq. (8). Thus the unsteady perturbation in the roll moment coefficient is obtained in the time-domain as:

$$
\Delta C_{l}(t)=A_{0} C_{l} \eta(t)+\frac{b}{U_{\infty}} A_{1} C_{l} \dot{\eta}(t)+\left(\frac{b}{U_{\infty}}\right)^{2} A_{2} C_{l} \ddot{\eta}(t)+D_{C_{l}} y_{a}(t)
$$

Remark 2.2: The major effect of antisymmetric deformation of the wing on the overall dynamics of the aircraft is due to the change in roll moment coefficient.

\subsection{Propulsion System Model}

The propulsion system model is based on the data for an F-100 turbofan engine installed in the F-15. The steady-state values for idle, military, and maximum afterburner thrust are tabulated as functions of Mach number, altitude, and power lever angle (PLA). The model includes first-order core dynamics, with a time constant computed via linear interpolation as a function of percent military thrust, Mach number, and altitude. The afterburner model includes sequencing logic to handle the transitions between afterburner stages. 


\subsection{Fatigue Crack Damage Model}

The objective of the damage mitigating control is to inhibit or reduce fatigue crack growth in the wing structure. Since the crack growth dynamics are highly nonlinear, they cannot be directly incorporated into the design of a linear controller. An example of this nonlinear behavior is the temporary retardation of crack growth following a stress overload. Under constant amplitude loading, a plastic zone of constant size exists at the crack tip. Following a stress overload, the plastic zone becomes larger within a few stress cycles but it takes many more cycles (e.g., in the order of thousands of cycles) to come back to the original size of the plastic zone after expiry of the overload pulse. While the stress overload itself may cause considerable crack growth during its tenure, the larger plastic zone inhibits or reduces crack growth rate over a prolonged period (many cycles) under the normal loading conditions that follow the overload. This phenomenon has been extensively studied based on experimental observations [Porter (1972) and Schjive (1976)] and analytical modeling by Newman (1992); Patankar et al., 1999]. The FASTRAN-II model (Newman, 1981, 1992) that is widely used in aerospace industry has been adopted in this paper for fatigue crack evaluation and prediction. The FASTRAN-II model is capable of capturing the effects of crack retardation under variable-amplitude loading.

For any maneuver involving lateral motion of the aircraft, the stress profiles from the left and right wings are different. It is assumed that, on the average, a pilot would fly equal numbers of left turns and right turns, and similarly would not favor any particular direction for other maneuvers. For this reason, when evaluating crack growth, the stress profiles from the left and right wings were strung together to form one block of cyclic stress profile. Thus, crack growth results apply equally to both wings.

\subsection{Damage-Mitigating Control System Model}

Figure 5 shows a schematic representation of the damage-mitigating control system in which the controller acts upon the subsystem models, described above, with the exception of the damage model that is placed outside the control loop solely for the purpose of damage evaluation. Since this paper focuses on robust linear control, it utilizes the information generated by a linear model within the control loop for prediction of fatigue crack damage. The nonlinear relationship between strain and fatigue crack growth rate is not directly handled by the controller synthesis procedure presented in this paper. Holmes and Ray (1998) have addressed this issue in a two-tier control architecture for damage-mitigating control of rocket engines in which the damage information is utilized for feedback control in real time.

\section{ROBUST CONTROLLER SYNTHESIS}

The $\mathrm{H}_{\infty}$-based structured singular value $(\mu)$ synthesis technique [Zhou et al., 1996] has been chosen as the robust controller design method in this paper. Since aircraft controllers are increasingly being implemented on digital computers, sampled-data control systems have been designed using the function $s d h f s y n$ in the MATLAB mutools toolbox [Balas et al., 1993] that is based on the methods of Bamieh and Pearson (1992) and Shivashanker 
and Khargonekar (1993). As mentioned earlier, separate robust controllers are designed for the lateral and longitudinal motion of the aircraft.

Next we describe the generalized plant models (i.e. the augmented system of the aircraft dynamics, actuator dynamics, plant modeling uncertainties, and performance weighting functions) used for synthesis of the longitudinal and lateral controllers, respectively. Note that the output of the crack-growth model is not used for feedback into the controller in either case. This paper focuses on robust control of flight dynamics in which the damage model is used only for analysis of the controller as well as for understanding the physical relationship between flight dynamics and fatigue crack growth. Nevertheless, the proposed control system has the flexibility of incorporating the output of the damage model as a feedback signal in an outer control loop following the architecture of Holmes and Ray (1998).

\subsection{Lateral Controller Synthesis}

The state vector used for synthesis of the lateral controller includes both the lateral rigid-body states and the states of the antisymmetric aeroelastic model. Since the spiral mode is not of interest during the controller synthesis phase, it is possible to neglect the role angle state without significantly altering the dynamics of the other modes. Thus, by considering the first three degrees of freedom for generalized displacements and eight additional aerodynamic states of the antisymmetric aeroelastic model in Eq. (8), the plant state vector of lateral motion becomes:

$$
x_{l a t}=\left[\begin{array}{llllllllllll}
p & r & \beta & \eta_{1} & \eta_{2} & \eta_{3} & \dot{\eta}_{1} & \dot{\eta}_{2} & \dot{\eta}_{3} & y_{a_{1}} & \cdots & y_{a_{8}}
\end{array}\right]^{T}
$$

where $\mathrm{p}$ is the roll rate;

$r$ is the yaw rate;

$\beta$ is the sideslip angle;

$\eta_{\mathrm{k}}, \mathrm{k}=1,2,3$ are the coefficients of the first three antisymmetric modes of structural deformation;

$\dot{\eta}_{k}, k=1,2,3$ are the time derivatives of $\eta_{k}, k=1,2,3$, respectively;

$\mathrm{y}_{\mathrm{a}_{\mathrm{k}}}, \mathrm{k}=1,2, \cdots, 8$ are the eight aerodynamic states chosen for the antisymmetric aeroelastic model.

The generalized plant used for synthesis of the lateral controller is shown in Figure 6. The ideal model contains two blocks; one is a first order system representing the desired roll mode time constant, and the other is a second order linear time-invariant system with the desired frequency and damping ratio of the Dutch roll mode. The performance weighting function $\mathrm{W}_{\mathrm{p}}(\mathrm{s})$ contains three blocks. The first two blocks penalize the differences in roll rate and sideslip responses of the aircraft model and the ideal model. The third block penalizes the difference in bending strain between the left and right wings. Although the wings are subjected to both bending and torsional displacements, the magnitude of the torsional strain is about two orders of magnitude lower than the bending strain. Thus, the principal strain is essentially equal to the bending strain, and only the bending strain is penalized during controller synthesis. However, both values are used as feedback signals for control purposes. The strain weighting functions are selected for the damage-mitigating controller design based on the information generated from 
extensive simulation runs. The transfer function $\mathrm{W}_{\mathrm{c}}(\mathrm{s})$ represents the frequency-dependent weights placed on the antisymmetric stabilator deflection and its rate. These weights are constant over the frequency range of interest, and are chosen to be the inverses of the maximum position and rate. The low-pass filter on the reference signal is included to make the D-matrix of the generalized plant zero, which is a requirement of the MATLAB function $s d h f s y n$ for sampled data controller design. The transfer function $\mathrm{W}_{\text {del }}(\mathrm{s})$ is the uncertainty weight. In an actual design case, it would be desirable to characterize the uncertainty in the plant model based on the known dynamic behavior of the aircraft, preferably from experimental data. In this case, since the model [Brumbaugh, 1991] does not represent any specific aircraft, no such data was available. Therefore, we have chosen an uncertainty weight of $\frac{20000(s+100)}{(s+2000)(s+10000)}$, which represents $\sim 10 \%$ uncertainty at low frequencies increasing to $\sim 200 \%$ uncertainty at high frequencies. For the lateral model it is found that good results can be obtained when the ideal models are also used as the low-pass filters on the reference signals. This choice slightly reduces the order of the generalized plant.

\subsection{Longitudinal Controller Synthesis}

The state vector used for synthesis of the longitudinal controller includes both the longitudinal rigid-body states and the states of the symmetric aeroelastic model. However, since the phugoid mode is not of interest, it is possible to ignore the velocity, altitude, and pitch angle states without significantly altering the short period response of the aircraft. Thus, by considering the first three degrees of freedom for generalized displacements and eight additional aerodynamic states of the symmetric aeroelastic model in Eq. (6), the plant state vector of longitudinal motion becomes:

$$
x_{\text {long }}=\left[\begin{array}{lllllllllll}
q & \propto & \xi_{1} & \xi_{2} & \xi_{3} & \dot{\xi_{1}} & \dot{\xi_{2}} & \dot{\xi_{3}} & x_{a_{1}} & \cdots & x_{a_{3}}
\end{array}\right]^{T}
$$

where $q$ is the pitch rate;

$\alpha$ is the angle of attack;

$\xi_{k}, k=1,2,3$ are the coefficients of the first three symmetric modes of structural deformation;

$\dot{\xi}_{\mathrm{k}}, \mathrm{k}=1,2,3$ are the time derivatives of $\xi_{\mathrm{k}}, \mathrm{k}=1,2,3$, respectively;

$\mathrm{x}_{\mathrm{a}_{\mathrm{k}}}, \mathrm{k}=1,2, \cdots, 8$ are the eight aerodynamic states chosen for the symmetric aeroelastic model.

The generalized plant used for synthesis of the longitudinal controller is shown in Figure 7. The ideal model is a second order linear time-invariant system with frequency and damping ratio selected to match the desired short period response of the aircraft. The performance weighting function $\mathrm{W}_{\mathrm{p}}(\mathrm{s})$ contains two blocks. The first block penalizes the difference in pitch rate response between the outputs of the aircraft model and the ideal model. The second block penalizes the average bending strain of the left and right wings. The frequency-dependent weight $\mathrm{W}_{\mathrm{c}}(\mathrm{s})$ penalizes the actuator positions and rates of the symmetric stabilator deflection and its rate. Similar to the lateral controller, these weights are constant over the frequency range of interest, and are chosen to be the inverses 
of the maximum position and rate. The transfer function $\mathrm{W}_{\text {del }}(\mathrm{s})$ is the uncertainty weight, which is the same as that used for the lateral controller design.

Remark 3.1: For synthesis of linear control laws, the performance optimization must be carried out in terms of measurable quantities whose behavior can be captured by a linear model. The far-field strains near the critical point of each wing have been chosen for this purpose.

Remark 3.2: Frequency-dependent weighting functions are chosen for penalizing the average strain and the difference in strain at the critical points of the left and right wings. Due to the nonlinear relationship between strain and fatigue crack growth rate, the desired forms of the weighting functions are not known a priori. The existing literature with the exception of Holmes and Ray (1998) does not address this issue of strain weighting. Unfortunately, Holmes and Ray have not considered the effects of crack retardation. Simulation results presented later in this paper show that ignoring the effects of crack retardation under stress transients may lead to significant errors.

\section{Evaluation of the Damage-Mitigating CONTROL SyStem}

There are a few issues that need to be addressed for evaluation of damage-mitigating capabilities of an aircraft controller. First, it is necessary to ensure that comparable rigid-body motions are executed with each simulation run. For example, it would not be meaningful to compare crack growth for one maneuver having a peak load factor of $8 \mathrm{~g}$ with that from a almost similar maneuver in which the peak load factor is, say, $7 \mathrm{~g}$. The second issue is how to compare the results of crack growth from different simulation runs with due consideration to the effects of variableamplitude cyclic stresses. The third issue is evaluation of crack growth due to multi-axial stresses resulting from combined actions of the lateral and longitudinal controllers. Although the lateral and longitudinal dynamics of the aircraft are very weakly coupled and the respective controllers are designed separately, the fatigue crack damage depends on the total stress at the crack tip, to which both the symmetric and antisymmetric aeroelastic models make contributions. The controller design procedure should be a three-step process from these perspectives:

Step\#1: Evaluate all longitudinal Damage-Mitigating Controllers while the aircraft simulation model is executed for purely longitudinal maneuvers. Alternatively, one could lift the restriction on pure longitudinal motion as long as the same lateral controller is used in all cases.

Step\#2: Evaluate all lateral Damage-Mitigating Controllers while the aircraft simulation model is executed for combined lateral-longitudinal maneuvers using the same longitudinal controller. Limiting the maneuvers to pure lateral motion would also be an option for aircraft that are not designed for aggressive maneuvers. For fighter aircraft, however, the average stresses experienced under pure lateral motion would most likely be too far below the maximum allowable stresses for any significant fatigue crack growth to be observed.

Step\#3: After selecting one or more potential candidates from each of the previous two steps, the candidate lateral and longitudinal Damage-Mitigating Controllers must be evaluated in pairs under combined lateral and longitudinal maneuvering. 
In this paper, however, no significant damage-mitigation is achieved with the longitudinal controller. Therefore, Step\#3 is deemed unnecessary for this aircraft. Furthermore, since the bending stresses dominate the torsional stresses, fatigue damage is calculated based on the assumption of uniaxial (bending) stresses.

\subsection{Evaluation of Fatigue Crack Damage}

The fatigue life of a structure depends not only on the applied load profile but also on the initial condition of the crack damage in the structure. Starting with the same initial crack length, differences in crack growth profiles from different simulation runs can therefore be attributed solely to the actions of different controllers. Comparisons could then be made between the different controllers based on the number of maneuvers required for the crack length to reach a specified final crack length. For any maneuver involving lateral motion of the aircraft, although the stress profiles from the left and right wings are different, crack growth results apply to both wings. Over a flight mission, the numbers of left turns and right turns are expected to be similar with no particular emphasis on the direction of maneuvers. Therefore, for evaluation of crack damage, the stress profiles from the left and right wings are strung together to form a single block of (variable-amplitude) cyclic load corresponding to one average maneuver. The results are applicable to either wing - left or right.

\subsection{Simulation Results under Damage Mitigating Control}

The longitudinal controller in the aircraft under consideration is provided with only one control input (i.e., symmetric stabilator deflection) for regulating both the rigid-body pitch rate and the average strain at the wing root. In addition, no direct control of the symmetric force distribution on the wing span is available as these forces can only be changed at the expense of the rigid-body motion that determines the aircraft performance. Since the requirements of handling qualities must be met at low frequencies (up to approximately $10 \mathrm{rad} / \mathrm{sec}$ ), it would be unreasonable to influence the strain response within this frequency range. Thus, the only available choice for a strain weighting function is a band-pass filter. Several different filters were examined, however none seemed to have any significant effect on fatigue crack growth reduction without any significant loss of performance. The strain response was dominated by low frequencies, and the controller was unable to influence what little highfrequency content there was, due to the strict pitch rate response requirements. In the aircraft under consideration, the longitudinal controller is not very effective for damage mitigation. Therefore, we concentrate on the lateral controller in the sequel.

Since the aircraft under consideration is equipped with both ailerons and stabilators for roll control, the lateral controllers have the ability, within limits, to independently influence both the rigid-body roll rate and the stress transients in the wing. This allows examination of various types of strain weighting functions in the third block of $\mathrm{W}_{\mathrm{p}}(\mathrm{s})$ in Figure 2. Three basic types of strain weighting functions are investigated: an all-pass filter (constant weight), a low-pass filter, and a band-pass filter. In order to create a baseline case, one controller, denoted as the Performance Controller (PC) is synthesized without using any strain feedback or any penalty on the strain response. 
Additional strain weighting functions are provided for each of the lateral Damage-Mitigating Controllers (DMCs). Both the PC and DMCs are synthesized using a linearized model of the flexible aircraft in level, unaccelerated flight at an altitude of $5000 \mathrm{ft}$ and a Mach number of 0.8 . The results from a typical maneuver are presented below.

The turn reversal maneuver is selected for evaluating the damage-mitigating capabilities of the lateral controllers. Starting from level flight, the aircraft is rolled into a $7.5 \mathrm{~g}$ turn to the right, then quickly reversed into an

$8 \mathrm{~g}$ turn to the left, and finally returned to straight and level flight. For this test case, one complete (i.e., $360^{\circ}$ ) roll is executed in the clockwise direction. The first three out of four plates in Figure 4 show the roll rate, pitch rate, and sideslip angle transients, respectively, for the aircraft performing the turn reversal motion under the influence of each of three lateral controllers - PC, two DMCs (i.e., DMC1, and DMC2) along with the respective reference signals. The pitch rate of all three controllers is very close to the reference signal because this motion is largely governed by the longitudinal controller. The roll rate response of $\mathrm{PC}$ is practically identical to that of $\mathrm{DMC} 1$ and is slightly superior to that of DMC2. The sideslip response of PC is slightly superior to that of DMC1 while DMC2 shows larger deviations in the sideslip angle. The fourth plate in Figure 4 compares the fatigue damage at the wing root $\mathrm{mm}$ under these three controllers. The fatigue damage in each case is computed by the FASTRAN II model (Newman, 1992) based on the number of maneuvers required for the crack length to reach $1 \mathrm{~mm}$ starting from an initial value of $0.1 \mathrm{~mm}$. It follows from Figure 4 that DMC1 and DMC2 increase the fatigue life of wings by a factor of approximately $140 \%$ and $200 \%$, respectively, over PC. Therefore, DMC1 yields $40 \%$ saving in fatigue life with no apparent of loss of performance while DMC2 provides $100 \%$ saving with noticeable increase in sideslip deviation.

\section{SUMMARY AND CONCLUSIONS}

This paper presents the synthesis of damage-mitigating controllers (DMCs) for high-performance tactical aircraft. Formulation of the control laws takes into consideration the impact of fatigue crack damage at critical points (e.g., wings) of aircraft structure, as well as the performance requirements. A flexible wing model is formulated using the finite element method, and the dominant mode shapes and natural frequencies are identified. The doublet-lattice method is employed to develop an unsteady flow model for computation of the time-dependent spatial aerodynamic loads acting on the wing due to rigid-body maneuvers and structural deformation. These two models are subsequently incorporated into a pre-existing nonlinear rigid-body model of aircraft flight-dynamics.

The DMCs are designed using the $H_{\infty}$-based $\mu$-synthesis method. In addition to penalizing the error between the ideal performance and the actual performance of the aircraft, frequency-dependent weights are also placed on the strain amplitude at the root of each wing. Using each controller in turn, the aircraft is put through an identical sequence of maneuvers, and the resulting stress profiles are analyzed using a model of fatigue crack growth that includes the effects of crack retardation resulting from stress overload. Comparisons are made to determine the the resulting crack growth at the wing root for different DMCs. The results of simulation experiments show that the DMCs yield significant savings in fatigue life of the wing structure while retaining the dynamic performance of the 
aircraft. Specifically, the strain feedback is used for lateral motion control that manipulates the actuators (i.e., ailerons and stabilators) to simultaneously achieve high performance and damage mitigation.

Two important conclusions can be drawn from this research. The first is that damage-mitigating control of high-performance aircraft is achievable when redundant control surfaces are present. For the particular aircraft used in this paper, only the lateral controller was able to significantly influence fatigue crack damage in the wing due to its ability to allocate roll commands between the ailerons and the stabilators. Significant results could also be achieved with a longitudinal controller designed for an aircraft that has multiple control surfaces for longitudinal motion (such as canards, stabilators, leading-edge flaps, trailing-edge flaps, and thrust vectoring nozzles). Such a controller would be able to independently alter (within limits) the total lift and total pitching moment acting on the aircraft, and thus influence stresses in both the fuselage and the wings. The second and perhaps the more important conclusion is that DMCs cannot be reliably synthesized when the analysis does not include adequate information on the dynamic behavior of the crack-growth process. Intuition would lead one to believe that reductions in peak stress will result in a reduction in damage.

Damage mitigating control is potentially capable of extending the life of existing aircraft with no significant loss of performance. The methodology can also be employed to the simultaneously design structural components and control systems for new aircraft, thus providing the structural engineers with more accurate information on the damage that critical components would experience in service. This information facilitates the design of less conservative structures, resulting in lighter-weight, higher-performance aircraft. The methodology can be extended to transport aircraft for both military and commercial applications.

The main application of this research is anticipated to be in the aircraft design phase, since this will allow the structural engineers and the control systems engineers to simultaneously converge to their individual goals of ensuring both performance and structural integrity of the aircraft. This approach will dramatically reduce the number of iterations required to arrive at a final design that can safely maximize the maneuvering capabilities of the aircraft.

\section{REFERENCES}

Adams, J.R., Buffington, J.M., Sparks, A.G., and Banda, S.S., 1994, Robust Multivariable Flight Control, SpringerVerlag, London.

Albano, E., and Rodden, W.P., 1969, "A Doublet-Lattice Method for Calculating Lift Distributions on Oscillating Surfaces in Subsonic Flows," AIAA Journal, Vol. 7, No. 2, pp. 279-285; also Errata, Vol.7, No. 11, p. 2192.

Anderson, T.L., 1995, Fracture Mechanics, Fundamentals and Applications, $2^{\text {nd }}$ ed., CRC Press, Boca Raton.

Balas, G.J., Doyle, J.C., Glover, K., Packard, A., and Smith, R., 1993, $\mu$-Analysis and Synthesis Toolbox, MUSYN Inc., and The Math Works, Inc.

Bamieh, B.A., and Pearson, J.B. Jr., 1992, "A General Framework for Linear Periodic Systems with Applications to $\mathrm{H}_{\infty}$ Sampled -Data Control," IEEE Transactions on Automatic Control, Vol. 37, No. 4, pp. 418-435.

Baldelli, D.H, Ohta, H., and Nitta, K., 1993, "Gust Load Alleviation of an Aeroelastic Wing Model," Transactions of the Japanese Society for Aeronautical and Space Sciences, Vol. 36, No. 113, pp. 125-142.

Brumbaugh, R.W., 1991, "An Aircraft Model for the AIAA Controls Design Challenge," AIAA Paper 91-2631.

Buffington, J.M., Sparks, A.G., and Banda, S.S., 1994, "Robust Longitudinal Axis Flight Control for an Aircraft with Thrust Vectoring," Automatica, Vol. 30, No. 10, pp. 1527-1540. 
Caplin., J., 1998, Damage-Mitigating Control of Aircraft for High Performance and Life Extension, Doctoral Dissertation in Mechanical Engineering, The Pennsylvania State University, University Park, PA.

DoD, 1980, Military Specifications - Flying Qualities of Piloted Airplanes, MIL-F-8785C, Department of Defense, Washington, D.C.

Dowell, E.H. (Editor), Crawley, E.F., Curtiss, H.C. Jr., Peters, D.A., Scanlan, R.H, and Sisto, F., 1995, A Modern Course in Aeroelasticity, Third Revised and Enlarged Edition, Kluwer Press, Dordrecht, The Netherlands.

Durham, W.C., 1992, "Constrained Control Allocation," Proceedings of the 1992 AIAA Guidance. Navigation, and Control Conference, pp. 1147-1155.

Etkin, B., 1972, Dynamics of Atmospheric Flight, John Wiley \& Sons, Inc., New York.

Holmes, M., and Ray, A., 1998, "Fuzzy Damage Mitigating Control of Mechanical Structures," ASME Journal of Dynamic Systems, Measurement and Control, Vol. 120, No. 2, pp. 249-256.

Karpel, M., 1990, "Time-Domain Aeroservoelastic Modeling Using Weighted Unsteady Aerodynamic Forces," Journal of Guidance, Control, and Dynamics, Vol. 13, No. 1, pp. 30-37.

Kallappa, P.T., Holmes, M., and Ray, A., 1997, "Life Extending Control of Fossil Power Plants for Structural Durability and High Performance," Automatica, Vol. 33, No. 6.

Matsuzaki, Y., Ueda, T., Miyazawa, Y., and Matsushita, H., 1989, "Gust Load Alleviation of a Transport-Type Wing: Test and Analysis," Journal of Aircraft, Vol. 26, No. 4, pp. 322-327.

McLean, D., 1990, Automatic Flight Control Systems, Prentice Hall International, London.

Newman, J.C., Jr., 1981, "A Crack-Closure Model for Predicting Fatigue Crack Growth under Aircraft Loading," Methods and Models for Predicting Fatigue Crack Growth under Random Loading, ASTM STP 748, pp. 53-84.

Newman, J.C., Jr., 1992, FASTRAN-II - A Fatigue Crack Growth Structural Analysis Program, NASA Technical Memorandum 104159, Langley Research Center, Hampton, VA.

R. Patankar, A. Ray and A. Lakhtakia, 1999, "A State-Space Model of Fatigue Crack Growth," International Journal of Fracture, Vol. 90, No.3, pp. 235-249.

Pierce, G.A., 1988, Potential Flow Solutions, The Georgia Institute of Technology, Atlanta, GA.

Porter, T.R., 1972, "Method of Analysis and Prediction for Variable Amplitude Fatigue Crack Growth," Engineering Fracture Mechanics, Vol. 4, pp. 717-736.

Ray, A., Wu, M-K., Carpino, M., and Lorenzo, C.F., 1994, "Damage-Mitigating Control of Mechanical Systems: Parts I and II," ASME Journal of Dynamic Systems, Measurement and Control, Vol. 116, No. 3, pp. 437-455.

Ray, A., Dai, X., Wu, M-K., Carpino, M., and Lorenzo, C.F., "Damage-Mitigating Control of a Reusable Rocket Engine," AIAA Journal of Propulsion and Power, Vol. 10, No. 2, March/April, 1994c, pp. 225-234.

Rozak, J.H., and Ray, A., 1997, "Robust Multivariable Control of Rotorcraft in Forward Flight," Journal of the American Helicopter Society, Vol. 42, No. 2, pp. 149-160.

Rozak, J.H., and Ray, A., 1998, "Robust Multivariable Control of Rotorcraft in Forward Flight: Impact of Bandwidth on Fatigue Life," Journal of the American Helicopter Society, Vol. 43, No. 3, pp. 195-201.

Schijve, J., 1976, "Observations on the Prediction of Fatigue Crack Growth Propagation Under Variable-Amplitude Loading," Fatigue Crack Growth Under Spectrum Loads, ASTM STP 595, pp. 3-23.

Sivashankar, N. and Khargonekar, P.P., 1993, "Robust Stability and Performance Analysis of Sampled-Data Systems," IEEE Transactions on Automatic Control, Vol. 38, No. 1, pp.58-69.

Tangirala, S., Holmes, M., Ray, A., and Carpino, M., 1998, "Life-Extending Control of Mechanical Structures: Experimental Verification of the Concept," Automatica, Vol. 34, No. 1, pp. 3-14.

Thomton, S.V., 1993, Reduction of Structural Loads Using Maneuver Load Control on the Advanced Fighter Technology Integration (AFTI)/F-1/1 Mission Adaptive Wing, NASA TM-4526.

US Standard Atmosphere, 1962, US Government Printing Office, Washington, D.C.

Zhang, H., and Ray, A., 1998, "Robust Damage-Mitigating Control of Mechanical Structures: Experimental Verification on a Test Apparatus," IMECE (ASME) Symposium on Dynamics, Vibration, Diagnostics and Control of Structures and Components with Manufacturing Defects and Damage, Anaheim, CA.

Zhou, K., Doyle, J.C., Glover, K., 1996, Robust and Optimal Control, Prentice-Hall, NJ. 


\section{LIST OF TABLES AND FIGURES}

Table I Dynamic Models of Actuators

Figure 1 Transformation from Vertical Axes to Body-Fixed Axes

Figure 2 Relationship Between Body-Fixed, Stability, and Wind Axes

Figure 3 First Three Symmetric Mode Shapes

Figure 4 First Three Antisymmetric Mode Shapes

Figure 5 Damage Mitigating Control System Schematic

Figure 6 Generalized Plant Model for Lateral Controller Design

Figure 7 Generalized Plant Model for Longitudinal Controller Design

Figure 8 Aircraft Performance and Damage under Turn Reversal Maneuver 
Table I Dynamic Models of Actuators

\begin{tabular}{|l|c|c|c|}
\hline $\begin{array}{l}\text { Control } \\
\text { Surface }\end{array}$ & Transfer Function & $\begin{array}{c}\text { Deflection } \\
\text { Limits (deg) }\end{array}$ & $\begin{array}{c}\text { Rate Limits } \\
(\mathrm{deg} / \mathrm{sec})\end{array}$ \\
\hline Aileron & $\frac{1.0}{\left(\frac{\mathrm{s}}{75.0}\right)^{2}+\left(\frac{2 \times 0.59}{75.0}\right) \mathrm{s}+1.0}$ & \pm 20 & \pm 100 \\
\hline Rudder & $\frac{1.0}{\left(\frac{\mathrm{s}}{72.0}\right)^{2}+\left(\frac{2 \times 0.69}{72.0}\right) \mathrm{s}+1.0}$ & \pm 30 & \pm 100 \\
\hline Stabilator & $\frac{\mathrm{s}}{\left(\frac{82.9}{2}\right)^{2}+\left(\frac{2 \times 0.068}{82.9}\right) \mathrm{s}+1.0}$ & $\pm 15 /-25$ & \pm 60 \\
& {$\left[\left(\frac{\mathrm{s}}{36.4}\right)^{2}+\left(\frac{2 \times 0.41}{36.4}\right) \mathrm{s}+1.0 \|\left(\frac{\mathrm{s}}{105.3}\right)^{2}+\left(\frac{2 \times 0.59}{105.3}\right) \mathrm{s}+1.0\right]$} & & \\
\hline
\end{tabular}




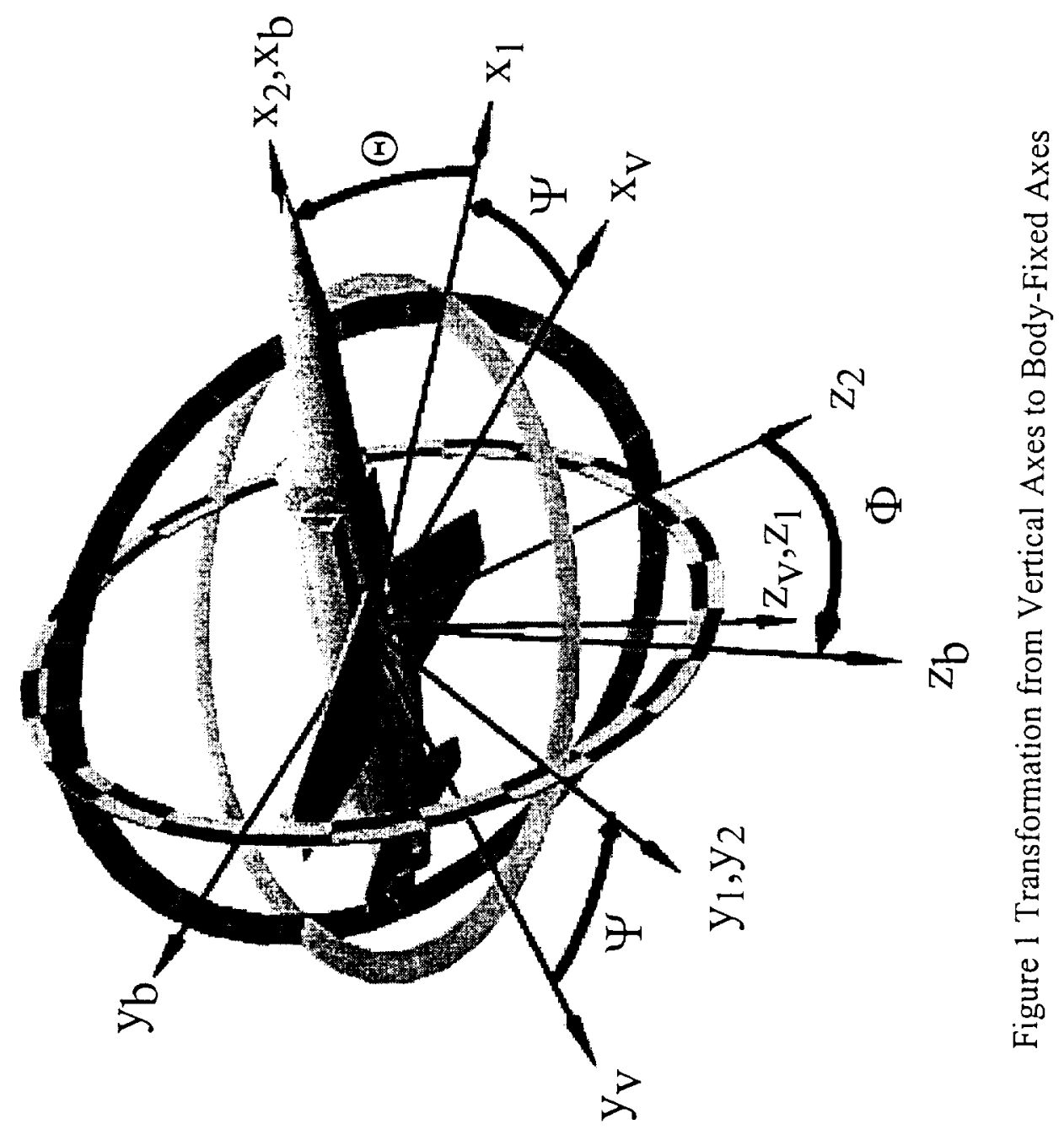




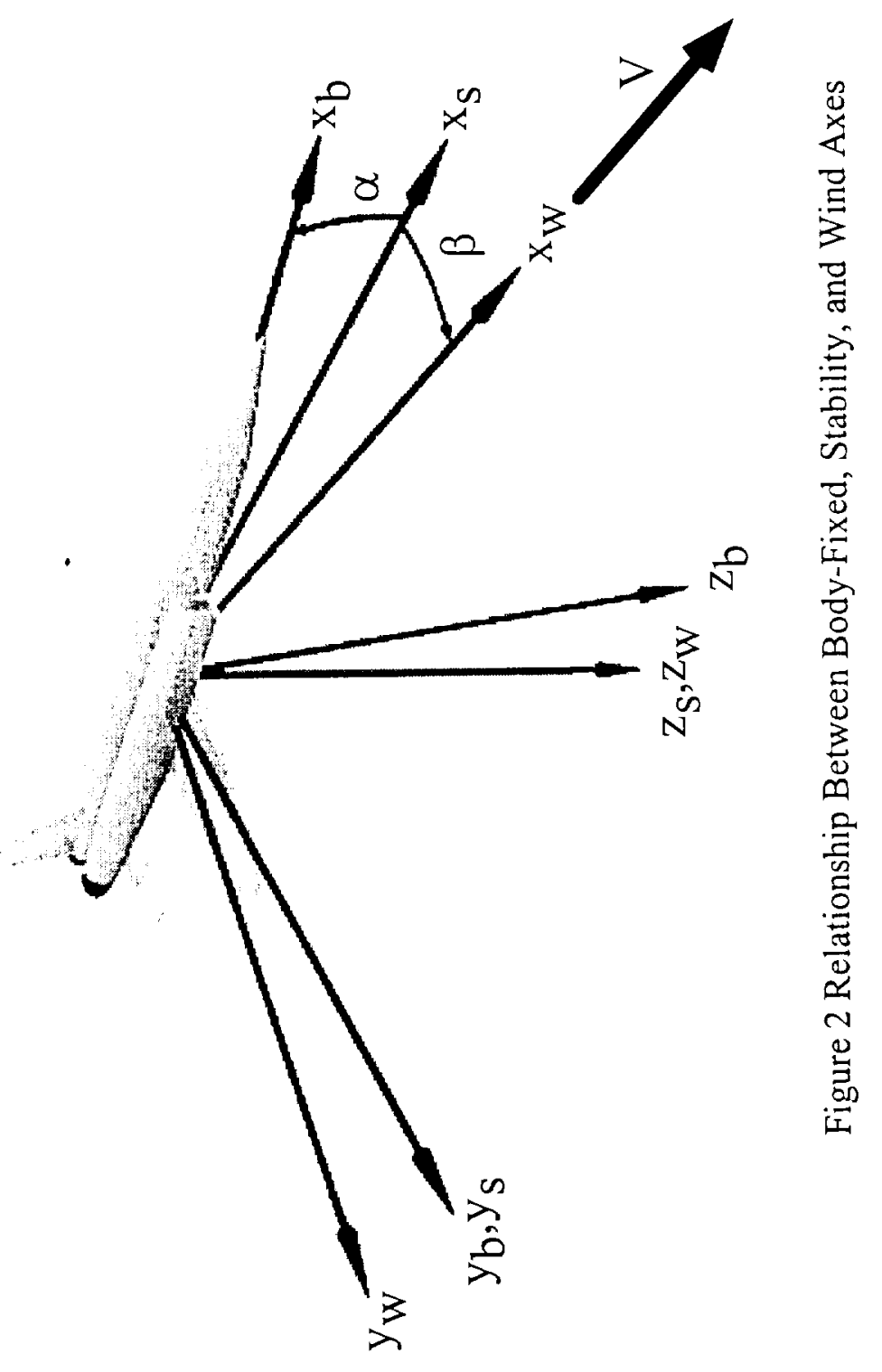




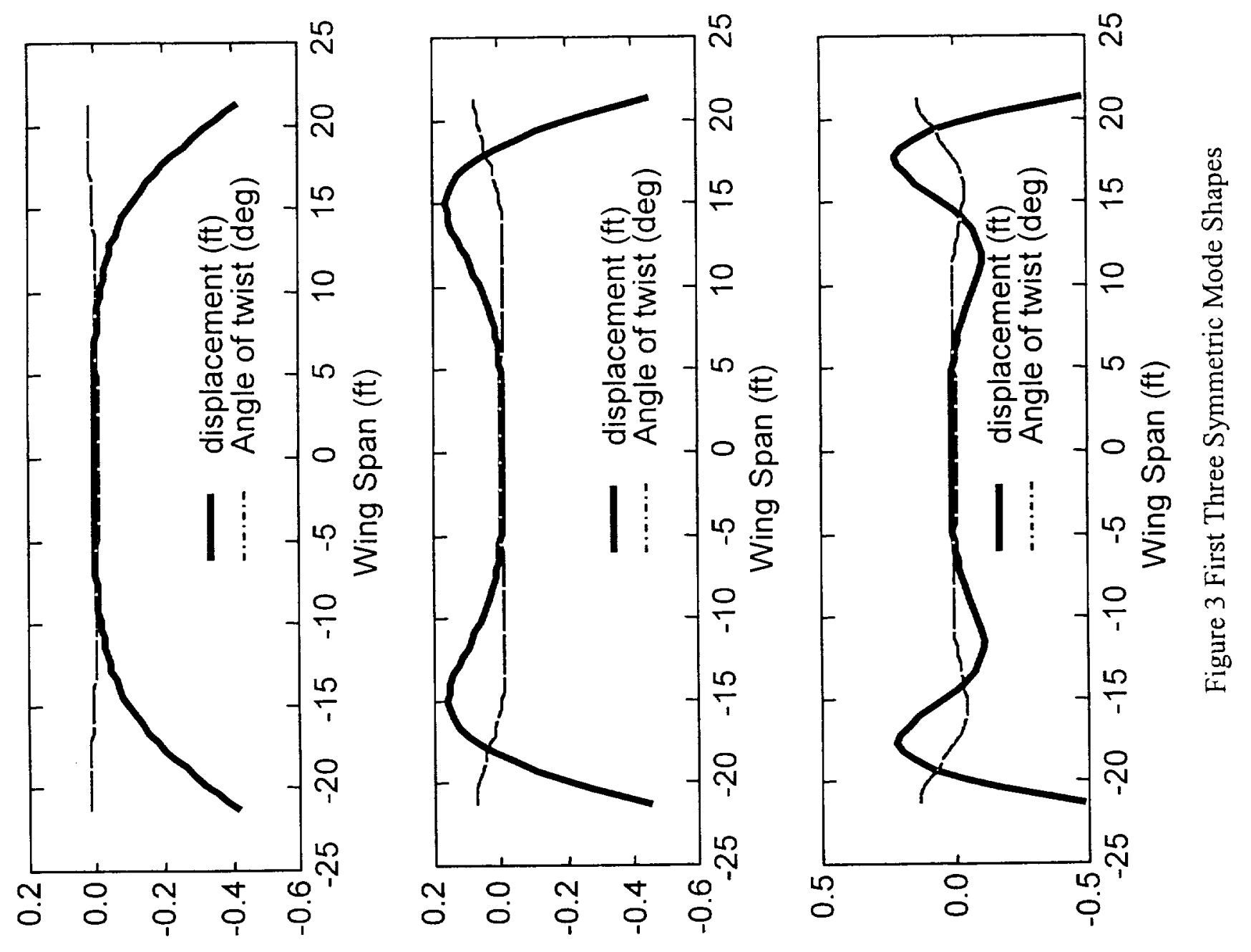




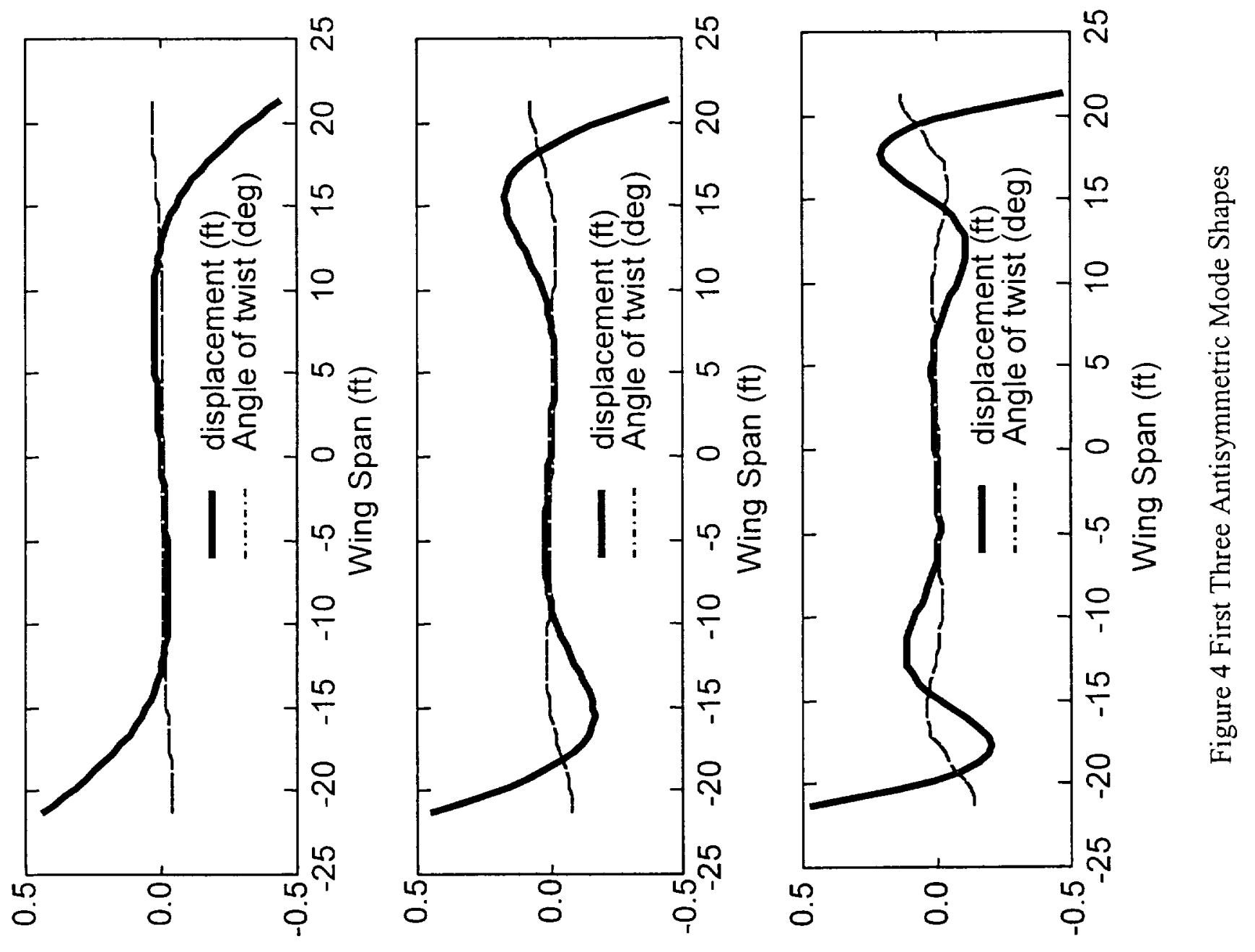




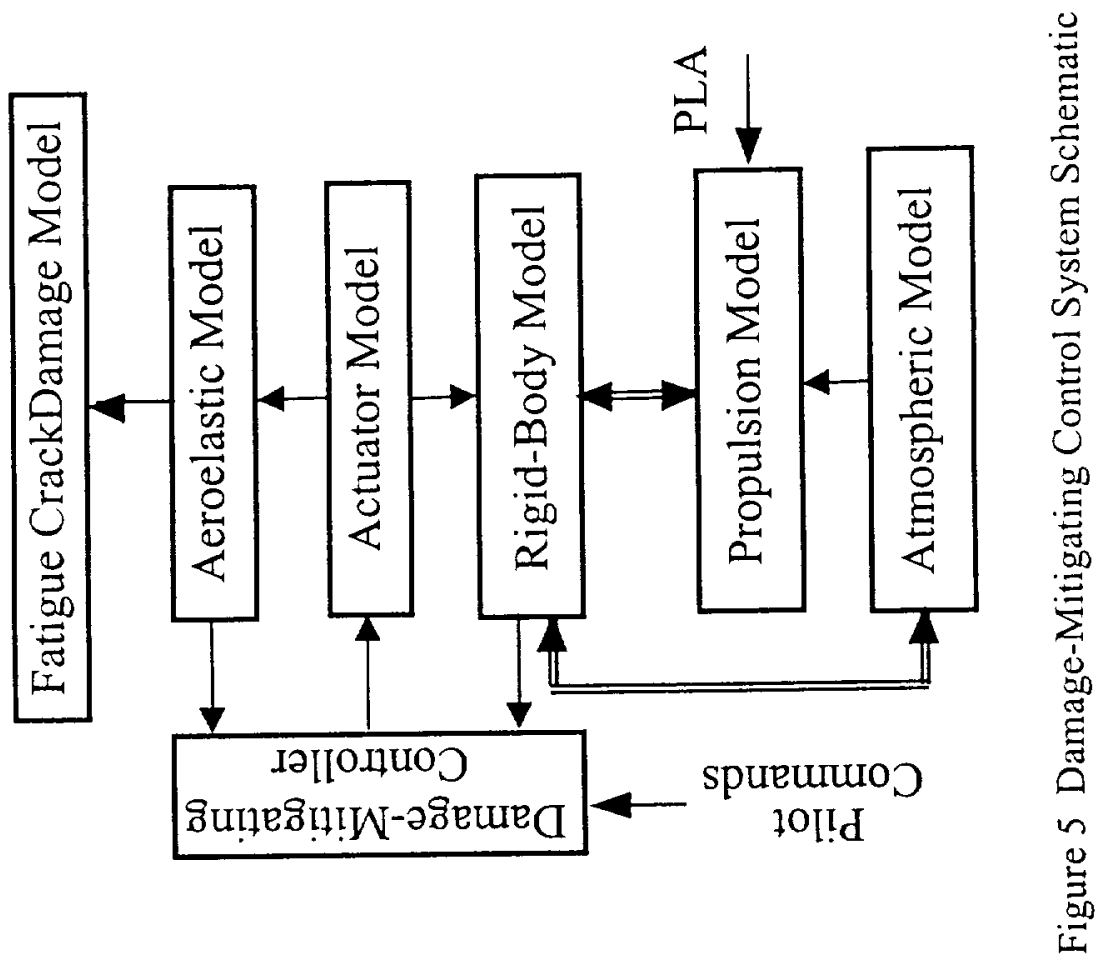




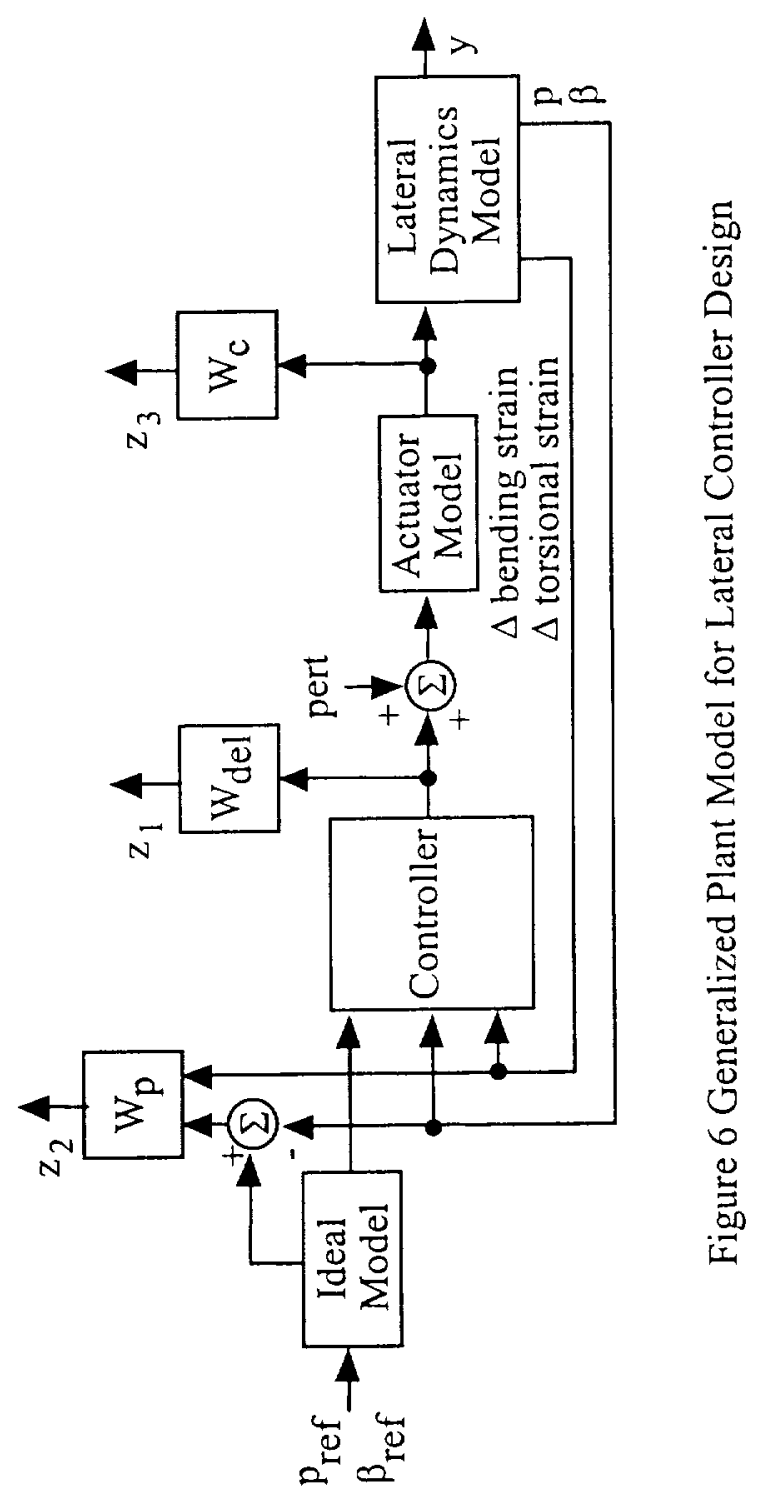




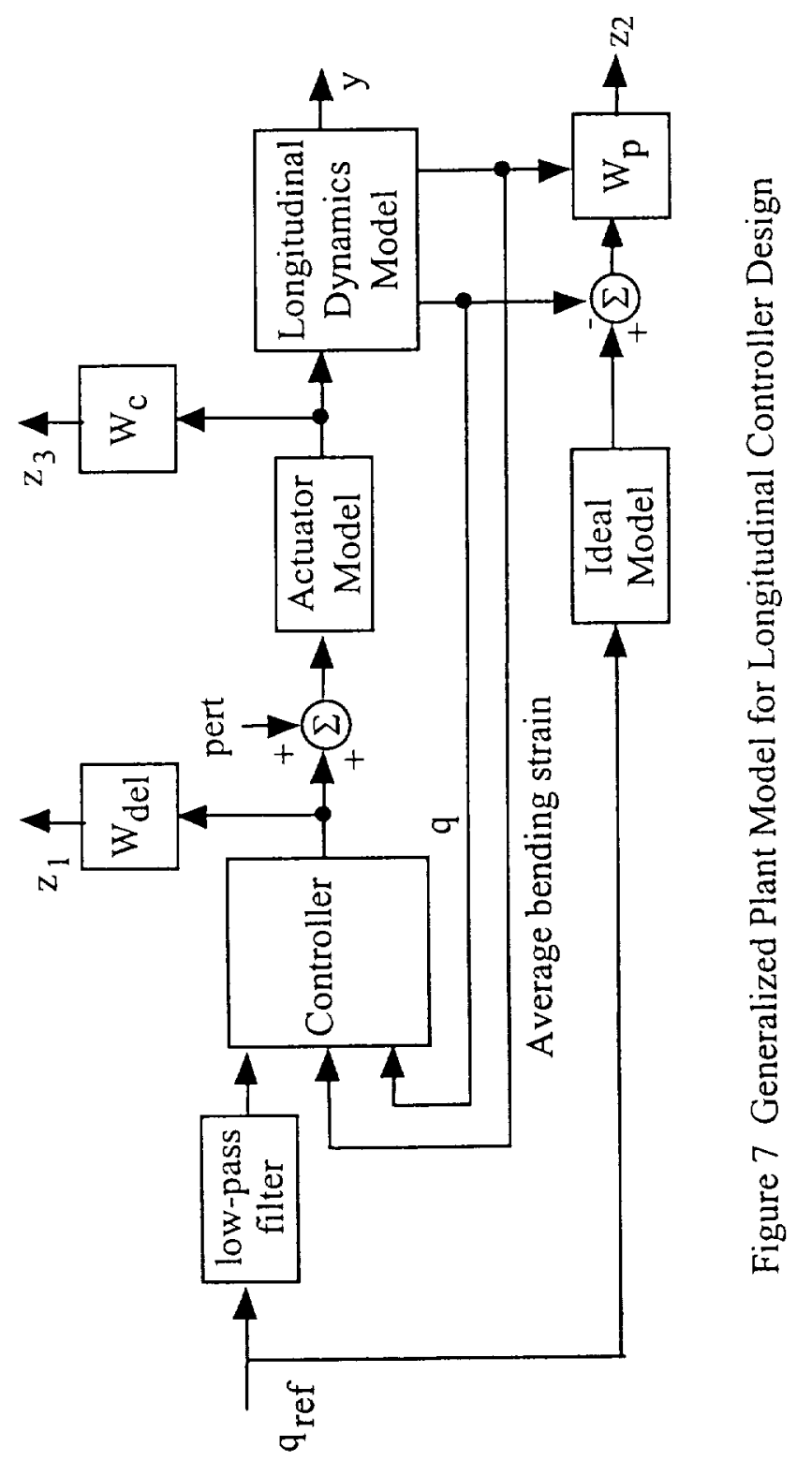



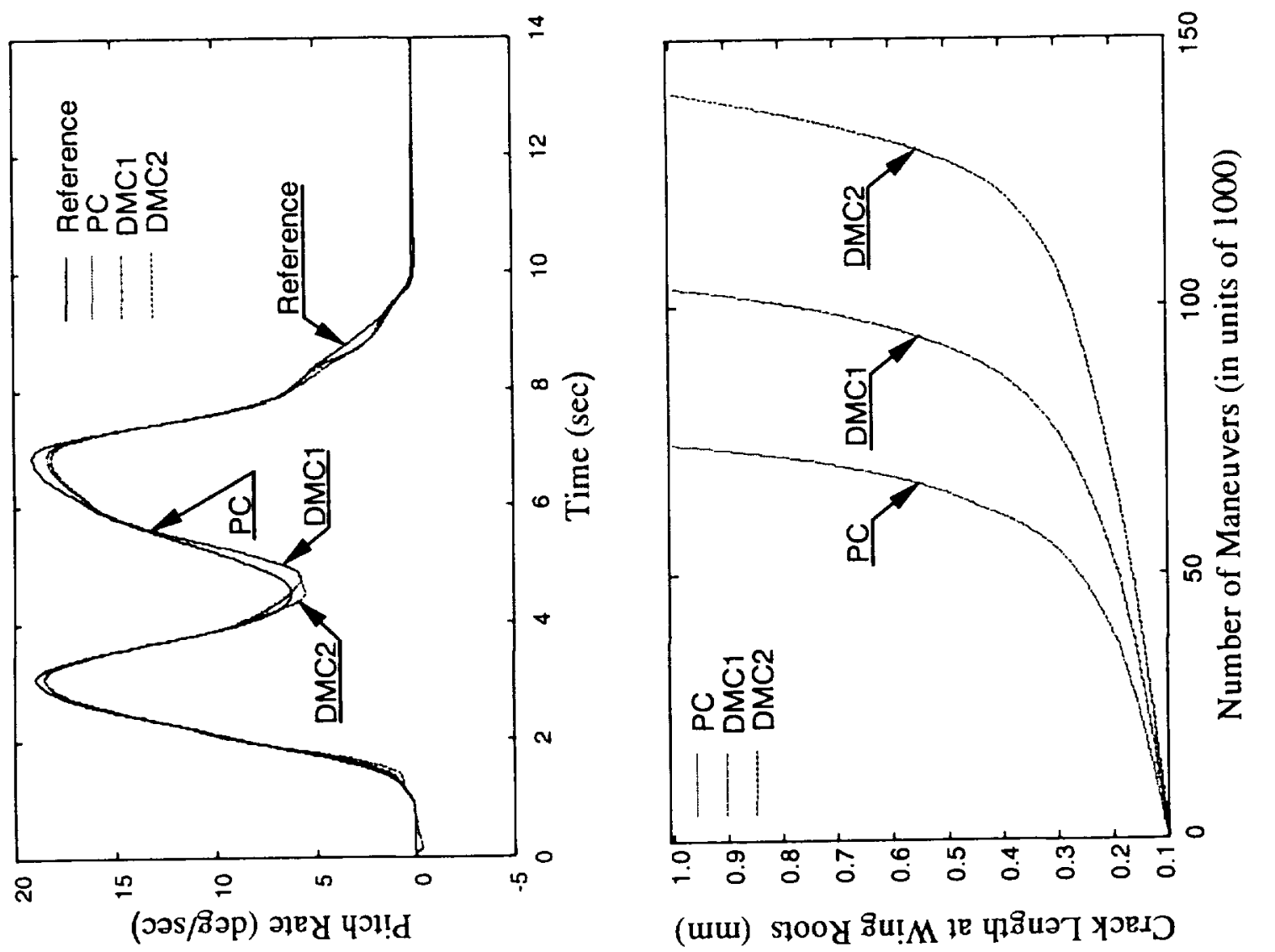

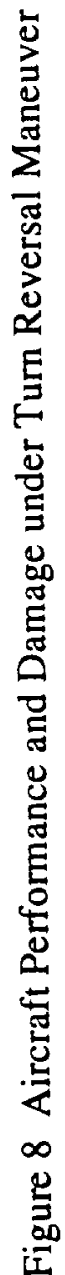
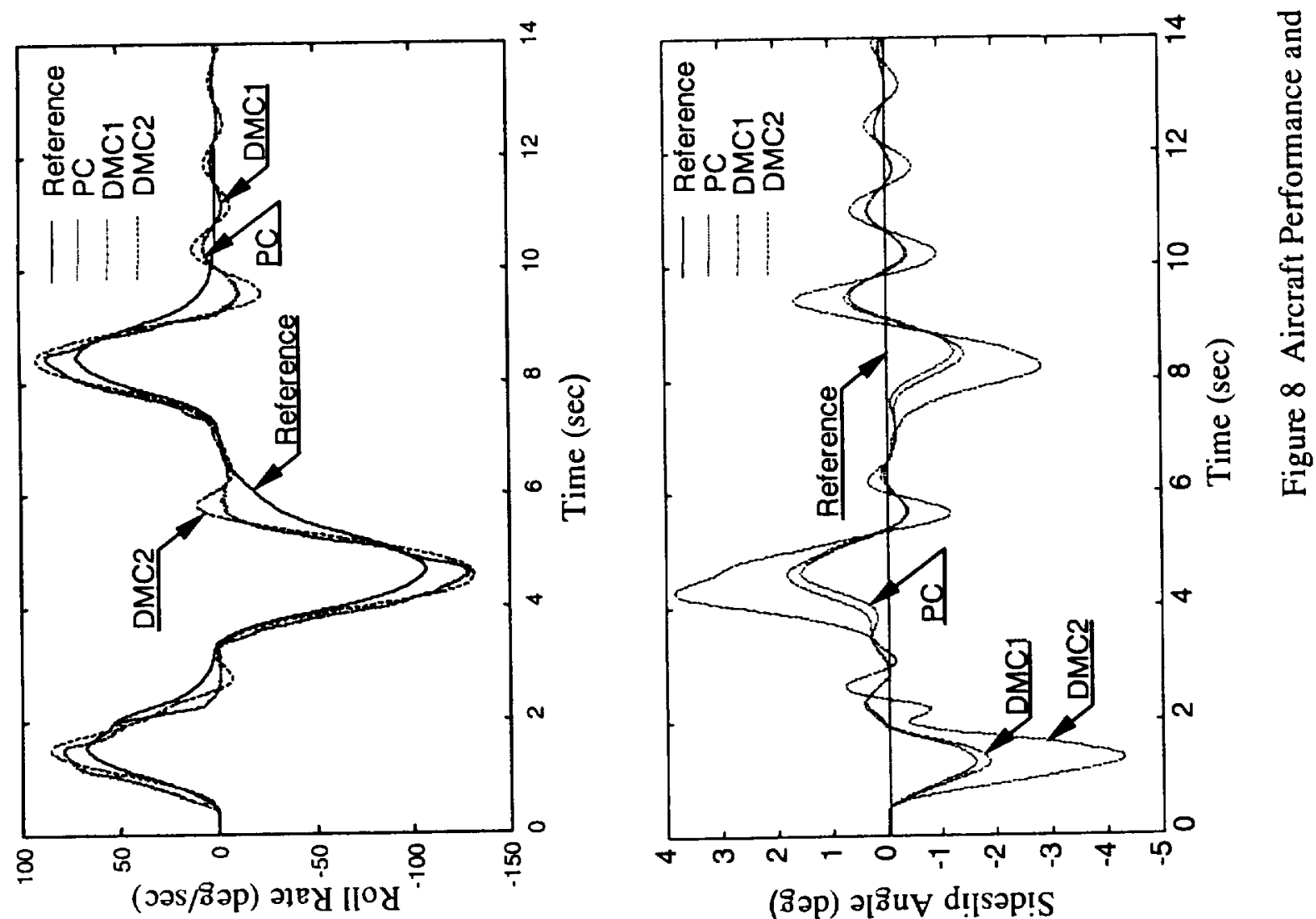